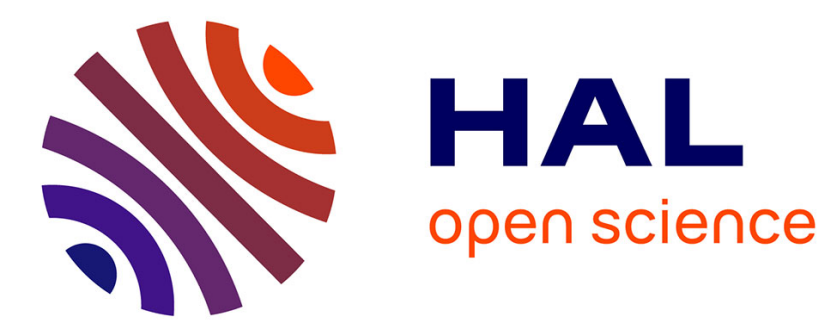

\title{
Magnetometers based on (double) relaxation oscillation SQUIDs
}

\author{
M. van Duuren, Y. Lee, D. Adelerhof, J. Flokstra, H. Rogalla
}

\section{To cite this version:}

M. van Duuren, Y. Lee, D. Adelerhof, J. Flokstra, H. Rogalla. Magnetometers based on (double) relaxation oscillation SQUIDs. Journal de Physique IV Proceedings, 1994, 04 (C6), pp.C6-255-C6260. 10.1051/jp4:1994641. jpa-00253137

\section{HAL Id: jpa-00253137 https://hal.science/jpa-00253137}

Submitted on 1 Jan 1994

HAL is a multi-disciplinary open access archive for the deposit and dissemination of scientific research documents, whether they are published or not. The documents may come from teaching and research institutions in France or abroad, or from public or private research centers.
L'archive ouverte pluridisciplinaire HAL, est destinée au dépôt et à la diffusion de documents scientifiques de niveau recherche, publiés ou non, émanant des établissements d'enseignement et de recherche français ou étrangers, des laboratoires publics ou privés. 


\title{
Magnetometers based on (double) relaxation oscillation SQUIDs
}

\author{
M.J. van Duuren, Y.H. Lee(1), D.J. Adelerhof, J. Flokstra and H. Rogalla \\ University of Twente, Dept. of Applied Physics, Low Temperature Group, P.O. Box 217, 7500 AE \\ Enschede, The Netherlands
}

\begin{abstract}
Relaxation Oscillation SQUIDs (ROS) and Double Relaxation Oscillation SQUIDs (DROS) combine the sensitivity of dc-SQUIDs with a large transfer, thus enabling flux locked loop operation with direct voltage readout by a room-temperature dc-amplifier, without flux modulation techniques. We fabricated several ROSs and DROSs with relaxation frequencies from 5 to $180 \mathrm{MHz}$. The experimental flux-to-frequency curves of the ROSs can well be fitted with theory, and the transfer (up to $170 \mathrm{MHz} / \Phi_{0}$ ) is large enough for practical use in a FLL without flux modulation. The experimental flux-to-voltage characteristics of the DROSs show very large transfer values of typically 10 to $30 \mathrm{mV} / \Phi_{0}$. A DROS operated in a simple FLL with direct voltage readout showed a white noise level of about $2 \mu \Phi_{0} / \sqrt{\mathrm{Hz}}$. A three channel DROS magnetometer system for biomagnetic measurements is under construction. In the DROSs that are to be used in this system, the signal flux is coupled to the SQUID by a tightly coupled 50-turn input coil.
\end{abstract}

\section{INTRODUCTION}

Conventional dc-SQUID magnetometers use non-hysteretic, shunted dc-SQUIDs. If a dc bias current is applied to the dc-SQUID, the voltage over the SQUID varies periodically with the applied magnetic flux, the maximum flux-to-voltage transfer $\partial \mathrm{V} / \partial \Phi$ is typically $100 \mu \mathrm{V} / \Phi_{0}[1]$, where $\Phi_{0}\left(=2.07 \cdot 10^{-15} \mathrm{~Wb}\right)$ is the flux quantum. Because of the very non-linear V- $\Phi$ curve, in most applications, the SQUID is operated in a feedback loop, known as Flux Locked Loop (FLL) in order to linearize the output signal.

The intrinsic (white) noise level of a shunted dc-SQUTD, mainly caused by thermal noise in the shunt resistors, is typically below $1 \mu \Phi_{0} / \mathrm{JHz}^{[1]}$. With a transfer of $100 \mu \mathrm{V} / \Phi_{0}$, this gives an output voltage noise of $1 \mu \Phi_{0} / \sqrt{\mathrm{Hz}} \cdot 100 \mu \mathrm{V} / \Phi_{0}=0.1 \mathrm{nV} / \sqrt{\mathrm{Hz}}$. However, the input voltage noise level of low-noise, room-temperature dc-amplifiers is one order of magnitude larger, so that direct readout of the dc-SQUID voltage by a room temperature amplifier limits the attainable noise level considerably. In practice, this problem is solved by the use of a lock-in amplification technique: a $100 \mathrm{kHz}$ modulation with an amplitude of $\Phi_{0} / 4$ is added to the feedback flux, which enables to increase the amplitude of the SQUID output voltage with a resonant transformer. This technique has shown to be very useful in practice, but nevertheless has some main disadvantages in multi-channel systems, where several SQUIDs are located close to each other. Some examples are crosstalk, narrow bandwidth, and the complexity of the system. This explains the interest of developing a second generation dc-SQUID, with a much larger transfer: these SQUIDs would enable FLL operation using direct readout, without flux modulation. 
Several new types of dc-SQUIDs have been presented to achieve higher transfers, for instance the SQUID with Additional Positive Feedback (APF) [2] and the digital SQUID [3]. We use Reloxation Oscillation SQUIDs and Double Relaxation Oscillation SQUIDs for realizing large transfers.

\section{2 (DOUBLE) RELAXATION OSCILLATION SQUIDS}

\subsection{The Relaxation Oscillation SQUID}

A ROS consists of a hysteretic dc-SQUID that is shunted by an inductor $\mathrm{L}_{\text {load }}$ and a resistor $\mathrm{R}_{\text {load }}$ in series, as shown schematically in fig. la. Fig. $1 \mathrm{~b}$ gives a schematic I-V curve of a hysteretic dc-SQUID. The critical current $I_{c}$ of the dc-SQUID depends periodically (with period $\Phi_{0}$ ) on the applied flux. $V_{g}$, the gap-voltage, is about $2.8 \mathrm{mV}$ for the $\mathrm{Nb} / \mathrm{Al}, \mathrm{AlO} \mathrm{x}_{\mathrm{x}} / \mathrm{Al} / \mathrm{Nb}$ Josephson junctions that we use.

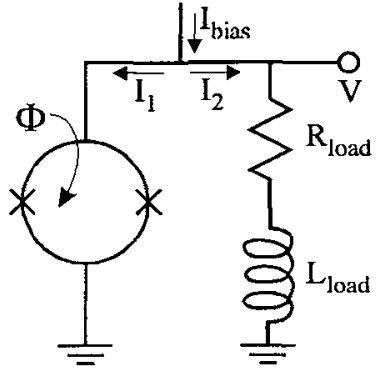

Figure 1a

Schematic diagram of a ROS.

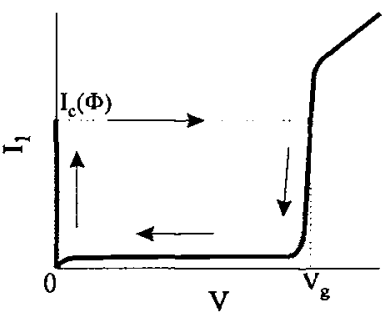

Figure $1 b$

Schematic I-V curve of a hysteretic dc-SQUID.

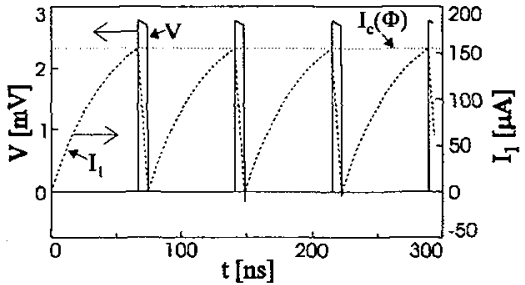

Figure lc

Numerical simulation of relaxation oscillations in a ROS.

If the ROS is biased with a de bias current, $I_{\text {bias }}>I_{c}(\Phi)$, relaxation oscillations will occur if the values of $\mathrm{L}_{\text {load }}$ and $\mathrm{R}_{\text {load }}$ are within certain limits, that have been determined by numerical simulations [4]. In fig. 1c, the result of such a numerical simulation is shown. The path in the I-V curve of the dc-SQUID that is passed through during the relaxation oscillations is indicated by the arrows in fig. $1 \mathrm{~b}$. As can be seen, at the output of the ROS a series of voltage pulses is generated. The frequency of these pulses is determined by the time constant of the shunt circuit $\left(\tau=\mathrm{L}_{\text {load }} / R_{\text {load }}\right)$, $I_{\text {bias }}$, and $I_{c}(\Phi)$. So, at a constant bias current, the output frequency $\mathrm{f}_{\mathrm{RO}}$ of the ROS varies with the applied flux: a ROS is a flux-to-frequency converter. We fabricated several ROSs with time constants designed between 5 and 26 ns. Standard photolitography and thin film sputter techniques on thermally oxidized $\mathrm{Si}$ wafers were used. All patterning, except for the definition of the Josephson junctions, is done by lift-off. The $4 \times 4 \mu \mathrm{m}^{2} \mathrm{Nb} / \mathrm{Al}, \mathrm{AlO}_{\mathrm{x}} / \mathrm{Al} / \mathrm{Nb}$ Josephson junctions of the SQUID are defined by reactive ion etching in an $\mathrm{SF}_{6}$ plasma. The critical current of the junctions is about $70 \mu \mathrm{A}$, the capacity of each junction is $0.5 \mathrm{pF}$, resulting in a SQUID capacity of $1 \mathrm{pF}$ and a maximum SQUDD critical current of $140 \mu \mathrm{A}$.

On top of the washer type dc-SQUID, which has an inductance of $20 \mathrm{pH}$, a single-turn input coil is placed, in order to be able to apply a signal flux.

The shunt resistors are made of $\mathrm{ff}$-sputtered $\mathrm{Pd}$, whereas the shunt inductor $\mathrm{L}_{\text {load }}$ is formed by a planar, double-layer, low capacitance multi-turn $\mathrm{Nb}$ coil.

Experimental relaxation frequencies ranged from 5 to $180 \mathrm{MHz}$. In fig. 2a, an experimental frequency spectrum of a ROS is shown. The higher harmonics in the signal (we were able to measure up to six higher harmonic components) are caused by the pulsed shape of the ROS output signal. Fig. 2b shows experimental $f_{\mathrm{RO}}-\Phi$ curves for different bias currents. The solid lines represent theoretical fits. As can be seen, the experimental data can be described well by theory. The asymmetry in the $\mathrm{f}_{\mathrm{RO}}-\Phi$ curves is caused by a magnetic coupling $(\mathrm{M} \approx 8 \mathrm{pH})$ between the shunt inductor $\mathrm{L}_{\text {load }}$ and the SQUID-loop. 


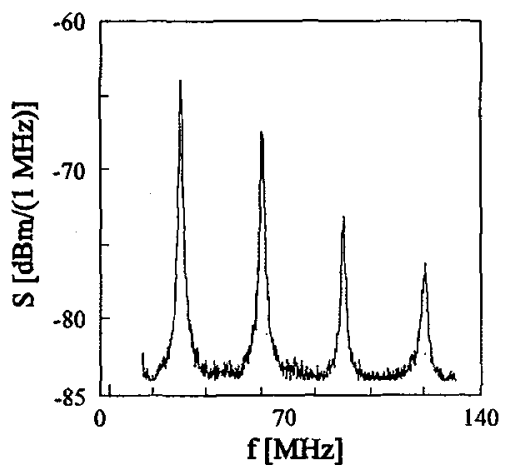

Figure 2a

Typical ROS output frequency spectrum.

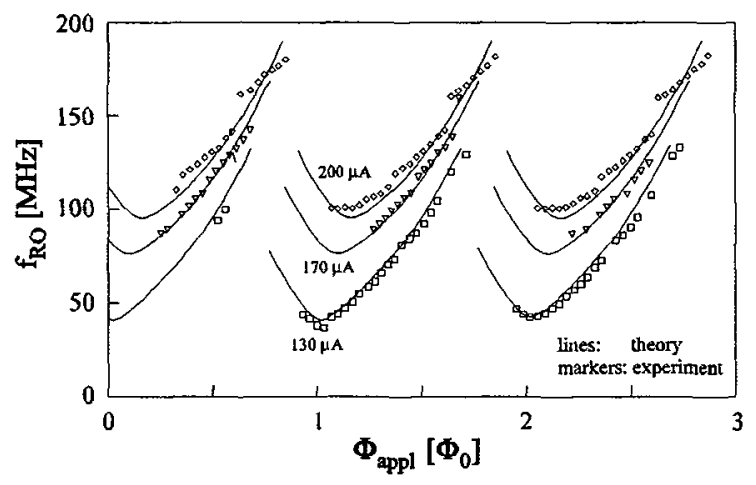

Figure $2 b$

Experimental $f_{R O^{-}} \Phi$ curve of a $R O S$.

FLL operation of a ROS with frequency readout is possible if a frequency-to-voltage converter is used. The flux-to-frequency transfer $\partial \mathrm{f}_{\mathrm{RO}} / \partial \Phi$ (about $\mathrm{f}_{\mathrm{RO}} / \Phi_{0}$ for all ROSs that have been characterized) is large enough to enable FLL operation without flux modulation.

\subsection{The Double Relaxation Oscillation SQUID}

\subsubsection{Principle of operation}

In fig. 3a, a schematic drawing of a DROS is given. The two SQUIDs are both hysteretic.

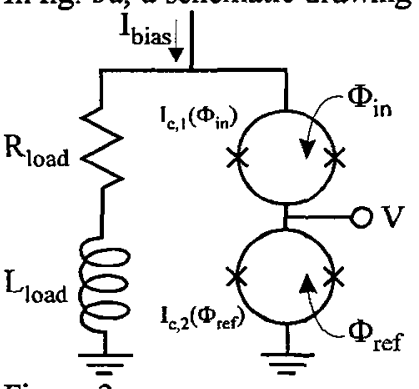

Figure $\overline{3} \mathbf{a}$

Schematic drawing of a DROS.

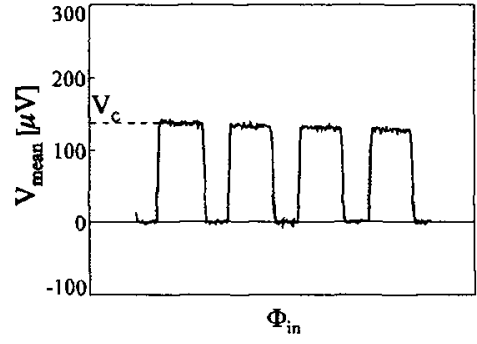

Figure 3b

Experimental $V-\Phi$ curve of a DROS.

The magnetic flux that is to be measured, $\Phi_{\mathrm{in}}$, is applied to the upper SQUID, the signal SQUID, while a constant reference flux, $\Phi_{\text {ref }}$, is applied to the lower SQUID, the reference SQUID. So, the critical current of the signal SQUID varies with the signal flux $\left(I_{c, 1}=I_{c, 1}\left(\Phi_{i n}\right)\right.$, whereas the critical current of the reference SQUID has a constant value, $I_{c, 2}=I_{c, 2}\left(\Phi_{\text {ref }}\right)$.

If a constant bias current is applied to the DROS, relaxation oscillations will occur, just like in the case of a simple ROS. In fact, the DROS acts as an $I_{c}$-comparator during the relaxation oscillations: only the SQUID with the smallest critical current switches to the voltage state. This means that, if $I_{c, 1}<I_{c, 2}$, there is no voltage at the output of the DROS, whereas, if $I_{c, 1}>I_{c, 2}$, there is a high frequency signal at the output. If this high frequency signal is read out with a dc-amplifier (with a bandwidth much smaller than the relaxation frequency), only the mean value of this ac signal is measured: $V_{\text {mean }}\left(I_{c, 1}>I_{c, 2}\right)=V_{c}>0$, and $\mathrm{V}_{\text {mean }}\left(\mathrm{I}_{\mathrm{c}, 1}<\mathrm{I}_{\mathrm{c}, 2}\right)=0$. The result is a very large transfer $\partial \mathrm{V}_{\text {mean }} / \partial \Phi_{\text {in }}$ at points where $\mathrm{I}_{\mathrm{c}, 1}\left(\boldsymbol{\Phi}_{\mathrm{in}}\right)=\mathrm{I}_{\mathrm{c}, 2}\left(\boldsymbol{\Phi}_{\mathrm{ref}}\right)$, as can be seen in the experimental V- $\Phi$ curve of fig. $3 b$. 
We fabricated and characterized several DROSs, following the same fabrication process as for the ROSs. The design values of $\tau_{\text {load }}$ and $I_{c, \max }$ are the same as for the fabricaied ROSs. Again, the signal flux $\boldsymbol{\Phi}_{\text {in }}$ is applied by a single-turn input coil on top of the SQUID washer, as is the reference flux.

Typical experimental transfer values for these DROSs range from $\partial V_{\text {mean }} / \partial \Phi_{\text {in }}=10$ to $30 \mathrm{mV} / \Phi_{0}$, which is two orders of magnitude larger than the transfer of conventional dc-SQUIDs, thus enabling FLL operation with direct room-temperature voltage readout. The main advantage of a DROS over a ROS is the fact that voltage readout is simpler to implement in practice than frequency readout.

\subsubsection{Noise in the Double Reloxation Oscillation SQUID}

Several noise sources can be distinguished in the DROS, like thermal noise on the critical current of the two SQUIDs, thermal noise in the shunt resistor and noise arising from random interactions between the relaxation oscillations and the Josephson oscillations in the junctions [5]. In a properly designed DROS, the first noise source, thermal fluctuations of the critical current, gives the major contribution to the overall flux noise. It decreases with increasing relaxation frequency, since the relaxation frequency determines the ' $\mathrm{I}_{\mathrm{c}}$ sampling rate'. In our DROSs, having relaxation frequencies around $100 \mathrm{MHz}$, the theoretical flux noise level due to thermal fluctuations in the junctions is about $1 \mu \Phi_{0} / \sqrt{\mathrm{Hz}}$. This noise level is less than one order of magnitude higher than the typical noise level of shunted dc-SQUIDs. At relaxation frequencies of around $1 \mathrm{GHz}$, the theoretical noise performance of DROSs is comparable to that of non-hysteretic dcSQUIDs. However, due to the much larger flux-to-voltage transfer of DROSs compared to conventional dc-SQUIDs, FLL operation of a DROS with direct voltage readout is possible without decreasing the sensitivity significantly, whereas conventional dc-SQUIDs need flux modulation to achieve the ultimate noise performance.

Special care should be taken to prevent high frequency noise to enter the DROS via the leads connecting it to the electronics (bias current, voltage readout, feedback flux and reference flux), because this high frequency noise could mix down with the relaxation frequency and the Josephson frequency towards low frequencies. Therefore, all leads to the DROS should be low-pass filtered (as near as possible to the DROS), twisted in pairs and sufficiently screened.

\subsubsection{FLL operation of a DROS with direct voltage readout}

We operated several of our DROSs in the simple FLL that is shown in fig. 4. To prevent mixed-down noise, all leads to and from the DROS are low pass filtered at $1 \mathrm{MHz}$ (filters are not included in fig. 4).

Due to the large transfer of the DROS, we measured a white flux noise level of about $2 \mu \Phi_{0} / \sqrt{\mathrm{Hz}}$, which is quite close to the theoretical value, and less than one order of magnitude larger than the noise in standard shunted dc-SQUIDs, despite the modest noise performance of the readout amplifier (white input voltage noise of about $5 \mathrm{nV} / \sqrt{\mathrm{Hz}}$ ).

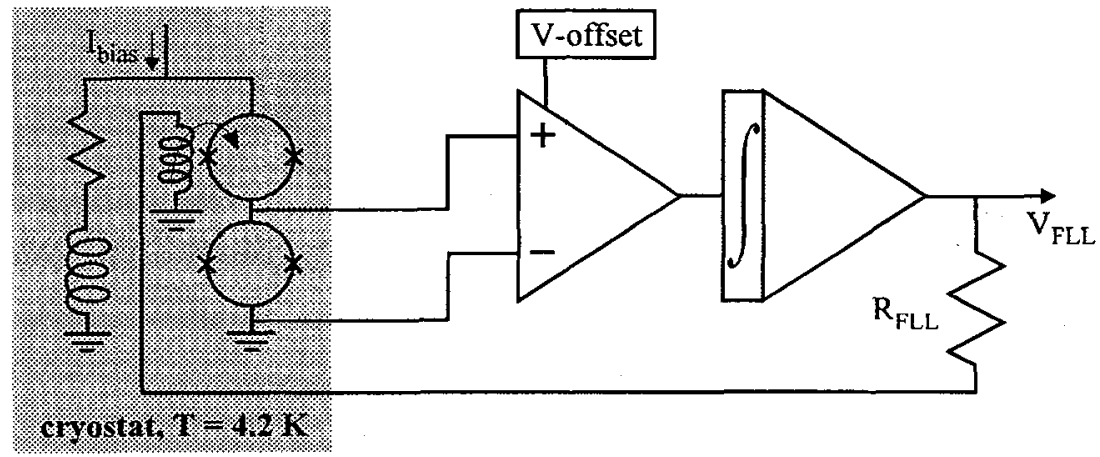

Figure 4

A DROS operated in a FLL based on direct voltage readout. 


\section{DEVELOPMENT OF A THREE-CHANNEL DROS MAGNETOMETER SYSTEM}

At this moment, a three-channel DROS magnetometer, to be used for biomagnetic experiments, is under construction in our group. In this system, 3 DROSs with integrated 50-turn input coils are coupled to three second order gradiometers. Several aspects of this magnetometer system are described below.

\subsection{Fabrication process and layout of the DROSs}

The DROSs for the three-channel magnetometer system are fabricated according to the fabrication process described in section 2 . The $\mathrm{Nb}$ washer type dc-SQUIDs are equipped with a tightly coupled 50-turn input coil, as introduced by Jaycox and Ketchen [6]. The sputtered $\mathrm{Nb}$ washers have a thickness of about $200 \mathrm{~nm}$, the diameter of the hole is 25 or $37.5 \mu \mathrm{m}$ for the signal SQUID, giving an inductance of 40 or $60 \mathrm{pH}$, respectively. The inductance of the reference SQUID is $40 \mathrm{pH}$ in all cases. The $\mathrm{Nb}$ input coil is insulated from the SQUID washer by $350 \mathrm{~nm}$ of $\mathrm{rf}$-sputtered $\mathrm{SiO}_{2}$. The final $\mathrm{Nb}$ layer is protected from oxidation by a thin ( $5 \mathrm{~nm}$ ) layer of $\mathrm{Pd}$.

The junction size is the same as for the (D)ROSs that have been discussed in section 2: $4 \times 4 \mu \mathrm{m}^{2}$. The critical current of the junctions is smaller: about $25 \mu \mathrm{A}$.

To minimize the magnetic coupling between the shunt circuit and the SQUIDs, the double-layer multi-turn shunt inductor is placed "far" away from the SQUIDs (about $1 \mathrm{~mm}$ ), and, moreover, the wiring of the shunt is designed symmetrically around the two SQUIDs. The time constant of the shunt circuit is about $10 \mathrm{~ns}$, resulting in a relaxation frequency of about $100 \mathrm{MHz}$.

\subsection{The flux transformer}

The inductance of the 50 turn input coil on top of the signal SQUDD is $100 \mathrm{nH}$ and $150 \mathrm{nH}$ for the $40 \mathrm{pH}$ and $60 \mathrm{pH}$ SQUID inductance, respectively. The input coil of each signal SQUID is connected to a niobium wire-wound pickup coil with a self-inductance of about $1.1 \mu \mathrm{H}$. To minimize the effect of external flux noise, the pickup coils are second order gradiometers. The diameter of the pickup coil is $19 \mathrm{~mm}$ and the base line is $22 \mathrm{~mm}$. The superconductive contact between the pickup coil and the input coil is achieved by ultrasonic bonding with $50 \mu \mathrm{m} \mathrm{Nb}$ wire [7]. In order to make the wire ductile enough for bonding, it is annealed under vacuum conditions. The thin Pd layer that covers the $\mathrm{Nb}$ contact pads of the DROSs to protect them from oxidation, has a positive influence on the quality of the superconductive bonding as well. The critical current of the superconducting bonds is measured to be more than $50 \mathrm{~mA}$, which is more than enough for our goal.

\subsection{The readout electronics}

Three identical electronic feedback systems have been constructed. Due to the large flux-to-voltage transfer of the DROS, the FLL electronics can be relatively simple; in principle the structure of the electronics is that of fig. 4. In the electronics for the three-channel system, we use LT1028 op-amps, which offer a lower voltage noise than the amplifier we used for the characterization of the DROSs with a single input coil: the white input voltage noise of our five-stage amplifier is $1.8 \mathrm{nV} / \mathrm{VHz}$. With a typical flux-to-voltage transfer of $10 \mathrm{mV} / \Phi_{0}$, the amplifier gives an equivalent flux noise of below $0.2 \mu \Phi_{0} / \sqrt{\mathrm{Hz}}$, much lower than the expected intrinsic noise level of the DROS (above $1 \mu \Phi_{0} / \sqrt{\mathrm{Hz}}$ for $\mathrm{f}_{\mathrm{RO}}=100 \mathrm{MHz}$ ). The gain of the amplifier can be adjusted continuously between $2 \cdot 10^{2}$ and $2 \cdot 10^{5}$, its bandwidth is larger than $1 \mathrm{MHz}$. The time constant of the integrator can be varied between $10^{-2}$ and $10^{-4} \mathrm{~s}$.

The electronics for each channel is placed inside a separate aluminium box, that is put directly on top of the cryostat, that is installed inside a magnetically shielded room. The A/D converters and micro-computer that are used for data-acquisition are placed outside the shielded room. 


\subsection{Layout of the system}

The insert is placed in a fibre glass He-cryostat, covered with aluminium foil to screen r.f. noise. The gradiometers on the base of the insert touch the bottom of the cryostat. Mechanical tensions due to thermal expansion of the cryostat and insert during cooling down are prevented because the gradiometers can move in axial direction. The leads from the gradiometers to the DROSs (about $10 \mathrm{~cm}$ ) are twisted, and shielded by a superconducting lead tube. The DROSs are magnetically shielded by a cylindrical compartmentalized $\mathrm{Nb}$ module in a $\mathrm{Pb}$ tube with an outer diameter of $10 \mathrm{~mm}$ and a length of $5 \mathrm{~cm}$.

Directly on top of the DROS module, an if filter module is connected that filters all leads going to the DROS. The simple first order low-pass RC-filters in this module have cut-off frequencies between 0.9 and $1.5 \mathrm{MHz}$, to allow a large measuring bandwidth. From the filter modules, phosphor bronze wires, twisted in pairs and shielded by a stainless steel pipe separately for each channel, go to the top of the cryostat, where they are connected to the three electronic boxes by a shielded 10-pins connector.

At this moment, the system is not fully completed, but all components (DROSs, electronics, cryostat wiring, filter modules, etc.) have been successfully tested. After completion of the system in the near future, the characterization of the system, and finally the biomagnetic experiments, can start.

\section{FUTURE DEVELOPMENTS}

The $100 \mathrm{MHz}$ DROSs used in the three channel system have a theoretical noise level of $2 \mu \Phi_{0} / \sqrt{\mathrm{Hz}}$, which is somewhat more than the typical noise level of shunted dc-SQUIDs. In order to increase the sensitivity of the DROS, the relaxation frequency has to be increased: at about $1 \mathrm{GHz}$, the theoretical noise level of about $0.6 \mu \Phi_{0} / \sqrt{ } \mathrm{Hz}$ (i.e. $\varepsilon \approx 20 \mathrm{~h}$ ) is comparable to the noise of standard dc-SQUIDs [5].

Another improvement of the present DROS design concerns the reference SQUID: since the critical current of this SQUID is tuned to a constant value, also a single junction, with a constant critical current somewhat below the maximum critical current of the signal SQUID, can be used. In this way, the number of wires going to the DROS can be decreased, which is very advantageous in multi-channel systems. We already fabricated some DROSs with a reference junction instead of a reference SQUID. However, probably due to resonance problems in the input coil, their V- $\Phi$ characteristics were somewhat irregular. A more detailed study of the interaction between the SQUID and its input coil is needed to solve this. Our final goal is the development of a "smart DROS" with integrated FLL, so that the complexity of the room-temperature readout electronics can be reduced further.

\section{CONCLUSION}

We fabricated ROSs and DROSs with different parameters. The experimental characteristics show a good correlation with theory. A three-channel DROS magnetometer system is under construction. A simple FLL without flux modulation techniques can be used for readout. Connected to wire-wound $\mathrm{Nb}$ second order gradiometers, the sensitivity of the system will be large enough for biomagnetic measurements.

\section{REFERENCES}

[1] Drung D., "Recent LTS SQUID Developments", EUCAS '93, Göttingen-FRG, Oct.4-8 (1993), 1287

[2] Drung D., Cantor R., Peters M., Scheer H.J. and Koch H., Appl. Phys. Lett. 57 (1990), 406

[3] Fujimaki N., FUJTSU Sci. Tech. J. 27-1 (1990), 59-83

[4] Adelerhof D.J., Nijstad H., Flokstra J. and Rogalla H., "(Double) Relaxation Oscillation SQUIDs with high $\partial V / \partial \Phi$, simulations and experiments", to be published in J. Appl. Phys.

[5] Adelerhof D.J., "Second Generation dc SQUTD magnetometers", thesis University of Twente (1993)

[6] Jaycox J.M. and Ketchen M.B., IEEE Trans. Magn. 17-1 (1981), 400-403

[7] Jaszczuk W., ter Brake H.J.M., Flokstra J., Veldhuis D., Stammis R. and Rogalla H., Measurement Science \& Technology 2 (1991), 1121-1122 